\title{
AMAZONITIZAÇÃO EM GRANITO RESULTANTE DA INTRUSÃO DE PEGMATITOS
}

\section{HANNA JORDT-EVANGELISTA ${ }^{1}$, JÚLIO CÉSAR-MENDES ${ }^{2} \&$ ANA LUÍSA CÔSSO LIMA ${ }^{2}$}

\begin{abstract}
AMAZONITIZATION IN GRANITE RESULTING FROM THE INTRUSION OF PEGMATITES Borrachudos-type granite ABSTRACT AMAZONITIZATION IN GRANITE RESULTING FIOM THE INTRUSION from Santa Maria do Itabira in Minas Gerais is cut by amazonite-bearing pegmatites, being marginally transformed into a green granite due to
amazonitization. This paper deals with the processes and the mineralogical and chemical variations involved in the transformation of the normal granite into a green granite.

The meter-wide pegmatite is composed of decimeter-large amazonite crystals of intense green color, cleavelandite, biotite, quartz and small The meter-wide pegmatite is composed of decimeter-large

The alkaline anorogenic granite is composed of quartz, nearly pure albite, microcline with triclinicities of 0.68 to 0.93 , Fe-biotite and accessory minerals. The mean $\mathrm{SiO}_{2}$ content is 74 weight $\%$, but in quartz-poor samples it decreases to $62 \%$. MgO is very low ( $\leq 0.05$ weight \%), minerals. The mean $\mathrm{SiO}_{2}$ content is 74 weight $\%$, but in quartz-poor samples it due to the retention of Eu in the calcic plagioclases left over in restitic rocks which generated the alkaline magma by partial melting.

The intensity of the green color in the granite increases towards the pegmatite contact. Chemically this color variation is accompanied by an increase in the $\mathrm{Pb}$ (and to a lesser extent also in $\mathrm{Rb}$ ) contents, while no difference could be registered for the major and other trace elements. $\mathrm{Pb}$ varies from $37 \mathrm{ppm}$ to $302 \mathrm{ppm}$, reaching $406 \mathrm{ppm}$ in the amazonite. It is possible that the subsolidus introduction of not only $\mathrm{Pb}$ but also $\mathrm{Pb}$ varies from $37 \mathrm{ppm}$ to $302 \mathrm{ppm}$, reaching $40 \mathrm{pas}$ responsible for the generation of $\mathrm{H}_{2} \mathrm{O}-\mathrm{Pb}$ pairs in the microcline, which are postulated of water from the pegmatite into the country rock was responsible for the considering the relatively low temperatures of pegmatitic magmas in the current literature as being the cause of the green color of amazonites. Considering the relatively since the aureoles locally reached a width equal to the width of the pegmatite.
\end{abstract}

Keywords: amazonite, metasomatism, lead, pegmatite, granite, Borrachudos, Santa Maria do Itabira.

RESUMO Granito do tipo Borrachudos de Santa Maria do Itabira, Minas Gerais, cortado por amazonita pegmatitos foi transformado marginalmente em granito verde em decorrência de amazonitização. Este trabalho trata dos processos e das variações mineralógicas e químicas en-

volvidos na transformação do granito normal em granito verde.
O pegmatito contém cristais de amazonita de tamanho decimétrico e intensa cor verde, cleavelandita, biotita, quartzo e pequenas quantidades de turmalina preta, galena, fluorita e anatásio.

O granito anorogênico alcalino é composto de quartzo, albita quase pura, microclina com triclinicidades variando de 0,68 a 0,93 , Fe-biotita e minerais acessórios. O teor de $\mathrm{SiO}_{2}$ médio é de $74 \% \mathrm{em}$ peso, mas em amostras empobrecidas em quartzo o teor cai para $62 \%$. MgO é muito minerais acessorios. $\mathrm{O}$ teor de $\mathrm{SiO}_{2}$ medio e de $7 \%$ em peso), $\mathrm{Fe} \mathrm{O}_{2}$ chega a $4,4 \%$ e os álcalis perfazem até $8,5 \%$ (em peso). $\mathrm{O}$ granito apresenta uma forte anomalia negativa

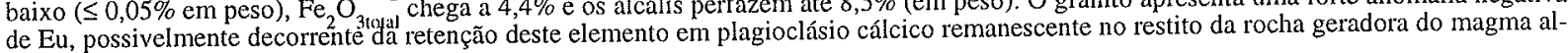
calino por fusão parcial.

A intensidade da cor verde no granito aumenta na direção do contato com o pegmatito. Quimicamente, esta variação de cor é acompanhada de um aumento no teor de $\mathrm{Pb}$ (e, em menor escala, também de $\mathrm{Rb}$ ), enquanto que não se registraram variações para os élementos maiores e outros um aumento no teor de $\mathrm{Pb}$ (e, em menor escala, também de $\mathrm{Rb}$ ), enquanto que náo se re $302 \mathrm{ppm}$ no tipo mais verde, chegando a 406 ppm na amazonita. É possível que a introdução, traços. $\mathrm{O} \mathrm{Pb}$ varia de $37 \mathrm{ppm}$ no granito branco ate $302 \mathrm{ppm}$ no tipo mais velde, chegando a 406 ppmán no estágio sub-sólido, de $\mathrm{Pb}$ e água na encaixante granítica durante a intrusão dos pegmatitos for responsácel pela geraçao de pares $\mathrm{H}_{2} \mathrm{O}-\mathrm{Pb}$. microclina, que são reportados na literatura como sendo a provável causa da cor verde em amazonitas. Considerando as temperaturas relativamente baixas de magmas pegmatíticos e a estrutura maciça do granito, o processo metassomático
eficiente, já que as auréolas alcançam localmente largura equivalente à do próprio pegmatito.

Palavras-chave: amazonita, metassomatismo, chumbo, pegmatito, granito, Borrachudos, Santa Maria do Itabira.

\section{INTRODUÇÃO}

Este trabalho versa sobre processos de amazonitização em rochas graníticas, ou melhor, de transformação de um granito normal, com feldspatos brancos, em granito verde portador de amazonita, em decorrência da intrusão de pegmatitos mineralizados em amazonita. São descritas as características mineralógicas e químicas das variedades branca e verde do granito, além de se abordar uma discussão sobre a origem da cor verde das rochas.

A região estudada está localizada no município de Santa Maria do Itabira (MG), na Fazenda Providência, $17 \mathrm{~km}$ a sul de Ferros (Fig. 1), onde ocorrem dois garimpos de amazonita em pegmatitos que cortam o granito Borrachudos. Este tipo de granito, definido originalmente por Dorr e Barbosa (1963) na região a NW de Itabira, aflora por uma região de 140 x $65 \mathrm{~km}$ de Santa Bárbara (a sul) até Sabinópolis (a norte) (Fernandes et al. 1995a,b). A idade do granito Borrachudos é Mesoproterozóica $(\sim 1,7 \mathrm{Ga}$, Dossin et. al. 1993). Trata-se de um granito anorogênico de afinidade alcalina, geneticamente relacionado à abertura do rift Espinhaço (Chemale Jr. 1987, Dossin et al. 1993, Fernandes et al. 1995b, Dussin et al. 1997). A geração dos pegmatitos da Província Pegmatítica Oriental de Minas Gerais, incluindo os do distrito pegmatítico de Santa Maria do Itabira, deu-se no ciclo Brasiliano, conforme sugerem datações de Bilal et al. (1995) em monazita (531 $\pm 22 \mathrm{Ma})$, bem como de Marciano et al. (1993) em moscovita $(519 \pm 10 \mathrm{Ma})$.

ROCHAS PORTADORAS DE AMAZONITA Dois corpos de amazonita pegmatitos vêm sendo explotados para peças de coleção e matéria prima para artesanato. A amazonita é de excelente qualidade por apresentar uma intensa cor verde a verde-azulada e por aparecer

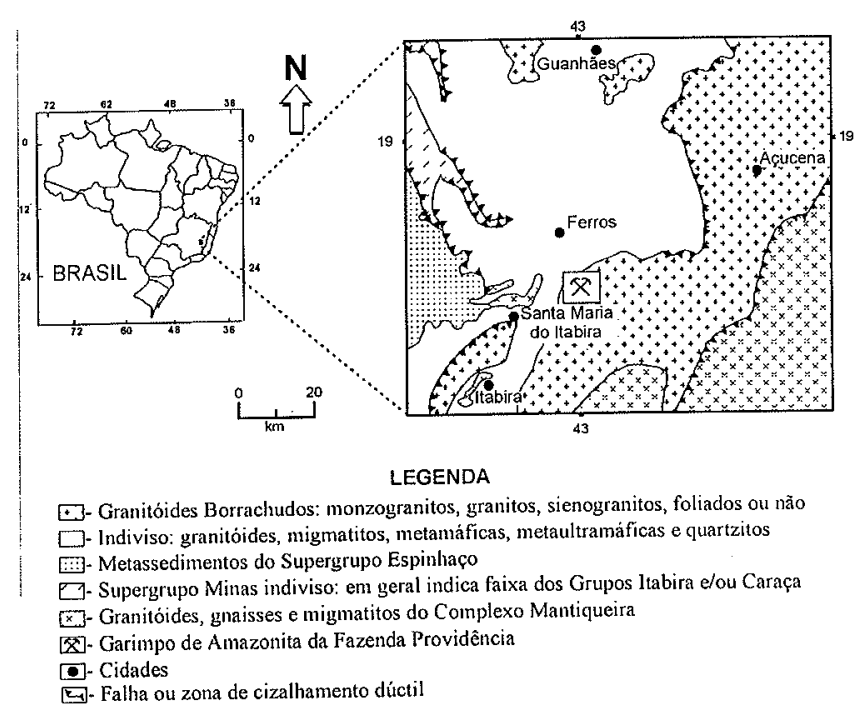

Figura I - Localização do Garimpo de Amazonita da Fazenda Providência (geologia simplificada de Pedrosa-Soares et al. 1994).

1 - Departamento de Geologia, Escola de Minas, Universidade Federal de Ouro Preto - Morro do Cruzeiro, s/n - 35.400-000 Ouro Preto - Minas Gerais - Brasil e-mail: hanna@degeo.ufop.br

2 - Idem, e-mail: julio@degeo.ufop.br

3- Bolsista de Iniciação Científica do CNPq, Departamento de Geologia, Escola de Minas, Universidade Federal de Ouro Preto 
em cristais até decimétricos muito bem formados. Os pegmatitos são tabulares, têm orientação geral NE e mergulhos de cerca de $55^{\circ}$ para $\mathrm{SE}$, com até $2 \mathrm{~m}$ de largura e ramificações de menor espessura. Os pegmatitos são discordantes em relação à foliação do granito e o seus contatos são abruptos. Xenólitos do granito dentro dos pegmatitos são comuns.

Os pegmatitos são do tipo simples e contêm, além da amazonita, cleavelandita incolor, biotita em placas que chegam a 3 decímetros de diâmetro e quartzo em intercrescimento gráfico com amazonita. Minerais acessórios são turmalina negra, fluorita, anatásio e galena. Esta última reflete o maior enriquecimento em $\mathrm{Pb}$ dos pegmatitos estudados no presente trabalho, em relação àqueles sem galena descritos por Marciano et al. (1994).

A litologia encaixante dos amazonita pegmatitos é o granito Borrachudos. A rocha tem textura granular subédrica média, com cristais de 2 a $3 \mathrm{~mm}$ em média. Quartzo e feldspato potássico podem atingir até $12 \mathrm{~mm}$. Os cristais de feldspato potássico e de albita são tipicamente euédricos em relação ao quartzo (Fig. 2). Uma incipiente foliação é conferida pela orientação preferencial planar de agregados lenticulares de biotita castanha, a qual constitui até $5 \%$ (em volume) da rocha. Quartzo, microclina e plagioclásio perfazem a quase totalidade da rocha ( $\rightarrow 0-95 \%$ em volume) e ocorrem em proporções semelhantes. De forma localizada, a percentagem de quartzo diminui e a rocha passa a ser quartzo sienito. O plagioclásio tem maclas polissintéticas do tipo albita e a sua composição é próxima da albita pura (Tabela 1). Os cristais de albita tendem a ser euédricos em relação ao quartzo (Fig. 2) e à microclina, em uma típica textura magmática. Não foram observadas, nas rochas estudadas, evidências texturais de substituição de plagioclásio por microclina conforme descritas em ocorrências deste granito em outros locais (Schorscher 1975, Machado 1998), sugerindo uma origem metamórfica-metassomática para o granito Borrachudos (Schorscher 1975). No caso presente, uma gênese por cristalização magmática é indicada.

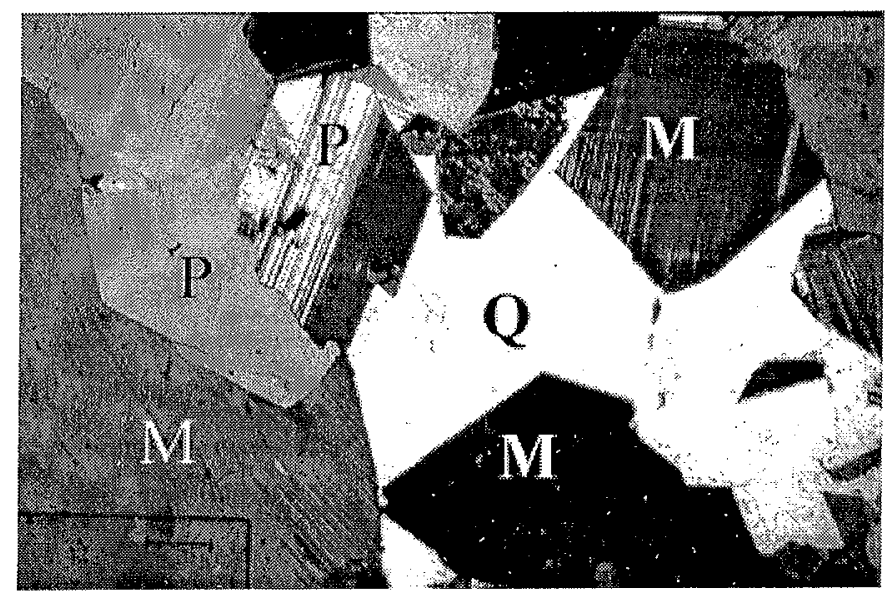

Figura 2 - Fotomicrografia da textura magmática do granito Borrachudos, mostrando plagioclásio $(P)$ e microclina $(M)$ euédricos e quartzo ( $Q)$ intersticial. Escala $=400 \mu m$, luz polarizada cruzada.

Os minerais acessórios são zircão, opacos (magnetita, ilmenita e sulfetos) e fluorita, a qual pode ser considerada como um mineral diagnóstico do granito Borrachudos mesmo quando este se apresenta gnaissificado devido a processos de milonitização. Hornblenda e allanita têm ocorrência local. Minerais de alteração/substituição ocorrem próximo aos pegmatitos, como clorita (em substituição parcial à biotita), mica branca (como sobrecrescimento de biotita) e mais raro carbonato (como preenchimento de fraturas).

Junto aos amazonita pegmatitos, os feldspatos brancos do granito Borrachudos adquirem cor verde, cuja intensidade diminui com a distância ao contato. A cor verde ocorre no feldspato potássico e a albita permanece com a cor branca. A auréola verde no granito tem largura variável, não ultrapassando 1 a 2 metros nos locais estudados. A auréola tem largura heterogênea, não permitindo sua explotação como rocha ornamental.

AMAZONITA É uma variedade de feldspato potássico de cor verde a verde-azulada. Na definição original de Breithaupt (1847) e se- gundo recomendação de Petrov et al. (1993) o feldspato é triclínico e, portanto, é uma variedade de microclina. No entanto, o nome amazonita vem sendo utilizado também para feldspatos potássicos do sistema monoclínico (ortoclásio) ou mesmo para feldspatos sódicos do tipo albita (Cech et al. 1971, Hofmeister \& Rossman 1985, Stevenson \& Martin 1986), desde que verde, verde-azulada ou azul.

A causa da cor na amazonita vem sendo motivo de polêmica desde Breithaupt (1847), o qual a atribuiu ao cromóforo $\mathrm{Cu}$. Outros cromóforos aventados (Petrov et al. 1993, Hofmeister \& Rossman 1983) são $\mathrm{Fe}, \mathrm{Cs}, \mathrm{Tl}, \mathrm{Rb}, \mathrm{Ba}$ e $\mathrm{Pb}$, além de defeitos estruturais. Atualmente há quase um consenso de que o $\mathrm{Pb}$ seria o elemento gerador da cor, pois amazonitas contêm teores muito maiores deste elemento (até $2 \%$ em peso, Stevenson \& Martin, 1986) do que as variedades brancas. No entanto, sabe-se que alguns feldspatos brancos também são enriquecidos em $\mathrm{Pb}$, ou que num mesmo cristal com irregular distribuição de cor, as porções verdes e as brancas apresentam teores similares daquele elemento (Stevenson \& Martin 1986). O íon $\mathrm{Pb}^{2+}$ não pode ser o causador da cor, pois a sua transição eletrônica ocorre na faixa do ultravioleta. No entanto, alguns estudos afirmam (Hofmeister \& Rossman 1983, 1985) que a cor pode ser causada pela presença de $\mathrm{Pb}$ em associação com água de cristalização. $\mathrm{Pb}$ bivalente e água substituiriam, num primeiro estágio, o K no feldspato:

$$
\mathrm{Pb}^{2+}+\mathrm{H}_{2} \mathrm{O}=2 \mathrm{~K}^{+} \text {. }
$$

A seguir a água sofreria uma dissociação radioativa causada por raios- $\gamma$ oriundos de ${ }^{40} \mathrm{~K}$, agindo como catalisadora tanto no processo de redução do $\mathrm{Pb}^{2+}$ para $\mathrm{Pb}^{1+}$ quanto de oxidação para $\mathrm{Pb}^{3+}$. A cor da amazonita é então atribuída por Hofmeister \& Rossman $(1983,1985)$ a centros de cor associados à transição eletrônica envolvendo $\mathrm{Pb}^{3+}$ (ou $\left.\mathrm{Pb}^{1+}\right)$. Neste caso a ausência de cor verde em feldspatos com altos teores de $\mathrm{Pb}$ pode ser atribuída a insuficientes quantidades de água na estrutura cristalina.

Em estudos mais recentes baseados em análises de EPR (ressonância paramagnética) Petrov et al. (1993) afirmam que a causa da cor em amazonita triclínica, isto é, em microclina verde, são centros de cor associados a pares $[\mathrm{Pb}-\mathrm{Pb}]^{3+}$. Como em outros tipos de feldspatos verdes de estrutura cristalina diferente (ortoclásio, albita) a causa da cor não poderia ser causada por estes pares, Petrov et al. (1993) sugerem que o termo amazonita seja restrito aos tipos triclínicos. No entender dos autores do presente trabalho, porém, esta restrição é inoportuna, pois implicaria em determinar-se primeiro a estrutura cristalina do mineral para então dar-se um nome às variedades verdes de feldspato, o que nem sempre é possível.

Nos pegmatitos e no granito da região estudada a amazonita ocorre como cristais com maclas em grade bem desenvolvidas e com abundante pertita na forma de venulações. Análises de difratometria de raios X mostram que a triclinicidade $\Delta$, calculada segundo a fórmula de Goldschmidt \& Laves (1954), varia de 0,68 a 0,93, indicando uma microclina do tipo intermediário a triclínica. Segundo Hofmeister \& Rossman (1985) o grau de ordem-desordem Al-Si exerce influência no comprimento de onda absorvido pela amazonita e, portanto, no matiz da cor. Eles mostram que amazonitas azuis são microclina s.s., isto é, possuem triclinicidade máxima $(\Delta \sim 1)$, enquanto que os tipos verdes são ortoclásio, com $\Delta \sim 0,6$ a 0,8 . Essa tendência foi confirmada nas amazonitas analisadas, pois as variedades com triclinicidades menores $(\Delta \sim 0,68$ a 0,85$)$, e que são encontradas nos pegmatitos, são verdes, enquanto a amazonita do granito, que possui triclinicidade muito elevada $(\Delta=0,93)$, é verde-azulada.

Análises de microssonda eletrônica foram realizadas em feldspatos (Tabela 1) de amostras selecionadas dos dois tipos de granito (normal e amazonitizado) e do pegmatito. Os dados de química mineral foram obtidos no Laboratório de Microssonda Eletrônica do Instituto de Geociências da Universidade de Brasília, num equipamento Cameca modelo SX 50 , operado a $15 \mathrm{kV}, 25 \mathrm{nA}$ e $10 \mathrm{~s}$.

Os feldspatos (Tabela 1) das variedades de granito (normal e verde) e do pegmatito são de dois tipos, feldspato potássico (microclina) e feldspato sódico (albita), cujas composições são, respectivamente, muito próximas às fórmulas teóricas dos componentes puros $\mathrm{Or}$ $\left(=\mathrm{KAlSi}_{3} \mathrm{O}_{8}\right)$ e $\mathrm{Ab}\left(=\mathrm{NaAlSi}_{3} \mathrm{O}_{8}\right.$ ). Em termos de elementos maiores, portanto, não há diferenças composicionais marcantes entre os feldspatos das diferentes litologias. A amazonita do pegmatito apresenta abundante pertita na forma de venulações, o que se reflete no seu teor de $\mathrm{Na}_{2} \mathrm{O}$ de quase $2 \%$ em peso (Tabela 2 , amostra AM). Nos re- 
Tabela 1 - Composição química (\% peso) e molecular (mol \%) média de feldspatos branco e verde do granito Borrachudos e da amazonita do pegmatito (n" de análises entre parênteses, $\sigma=$ desvio padrão).

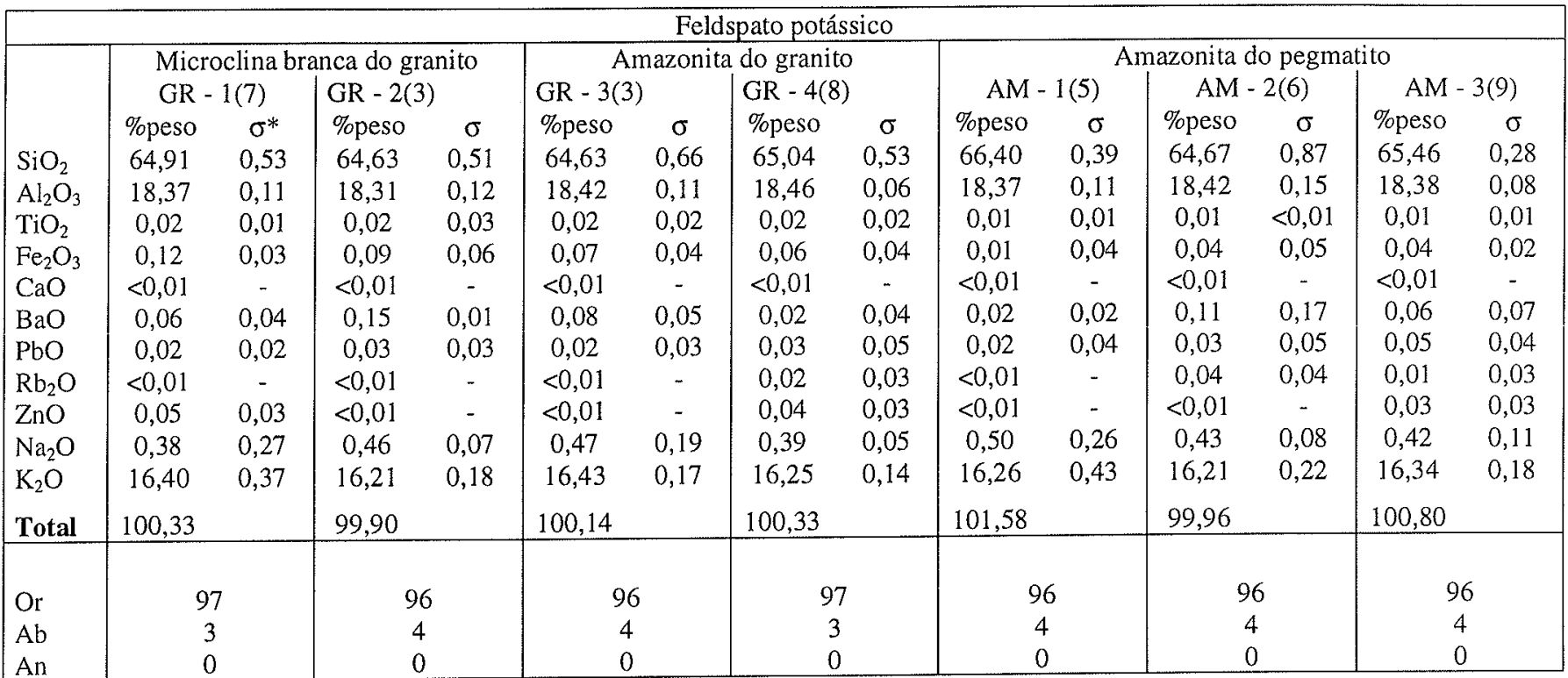

$* \sigma=$ desvio padrão

\begin{tabular}{|c|c|c|c|c|c|c|c|c|}
\hline \multicolumn{9}{|c|}{ Plagioclásio } \\
\hline & \multicolumn{2}{|c|}{ Granito branco } & \multicolumn{4}{|c|}{ Granito verde } & \multicolumn{2}{|c|}{ Pertita da amazonita } \\
\hline & GR - I(1) & $G R-2(1)$ & \multicolumn{2}{|c|}{ GR $-3(4)$} & \multicolumn{2}{|c|}{$\mathrm{GR}-4(3)$} & $\mathrm{AM}-2(1)$ & $\mathrm{AM}-3(1)$ \\
\hline & \%peso & \%peso & $\%$ peso & $\sigma^{*}$ & $\%$ peso & $\sigma$ & \%peso & \%peso \\
\hline & 67,21 & 68,81 & 68,87 & 0,75 & 68,51 & 1,21 & 68,82 & 68,69 \\
\hline $\mathrm{Al}_{2} \mathrm{O}_{3}$ & 19,69 & 19,46 & 19,60 & 0,34 & 19,50 & 0,03 & 19,61 & 19,66 \\
\hline $\mathrm{TiO}_{2}$ & $<0,01$ & $<0,01$ & $<0,01$ & - & $<0,01$ & - & $<0,01$ & 0,03 \\
\hline $\mathrm{Fe}_{2} \mathrm{O}_{3}$ & 0,05 & 0,07 & 0,06 & 0,03 & 0,03 & 0,02 & 0,08 & 0,09 \\
\hline $\mathrm{CaO}$ & 0,29 & 0,07 & 0,18 & 0,24 & 0,04 & 0,06 & 0,21 & 0,12 \\
\hline $\mathrm{BaO}$ & 0,00 & 0,00 & 0,01 & 0,02 & 0,00 & - & 0,00 & 0,02 \\
\hline $\mathrm{PbO}$ & $<0,01$ & $<0,01$ & 0,01 & 0,02 & $<0,01$ & - & $<0,01$ & 0,07 \\
\hline $\mathrm{Rb}_{2} \mathrm{O}$ & $<0,01$ & $<0,01$ & $<0,01$ & - & $<0,01$ & - & $<0,01$ & $<0,01$ \\
\hline $\mathrm{Na}_{2} \mathrm{O}$ & 10,11 & 10,54 & 10,58 & 0,20 & 10,48 & 0,12 & 10,27 & 10,42 \\
\hline $\mathrm{K}_{2} \mathrm{O}$ & 0,16 & 0,17 & 0,13 & 0,13 & 0,16 & 0,06 & 0,21 & 0,15 \\
\hline Total & 97,53 & 99,12 & 99,44 & & 98,72 & & 99,21 & 99,25 \\
\hline Or & 1 & 1 & & & & & 1 & 1 \\
\hline $\mathrm{Ab}$ & 96 & 98 & & & & & 97 & 98 \\
\hline An & 3 & 1 & & & & & 2 & 1 \\
\hline
\end{tabular}

$* \sigma=$ desvio padrão

sultados das análises de microssonda (Tabela 1) este teor aparece menor, pois as áreas selecionadas para análise não continham exsolução pertítica. Em termos de elementos-traço, verifica-se que os resultados analíticos mostram uma considerável dispersão indicada pelos desvios-padrão relativamente elevados, pois a microssonda eletrônica não é um equipamento adequado para análise de baixas concentrações. Apesar desta limitação, nota-se que o plagioclásio tende a ser empobrecido em $\mathrm{Ti}, \mathrm{Ba}, \mathrm{Rb}$ e $\mathrm{Pb}$ em relação ao feldspato potássico. Comparando o feldspato potássico branco com o verde e levando-se em conta os valores dos desvios-padrão, verifica-se uma tendência do verde ser mais enriquecido em $\mathrm{Pb}$ e $\mathrm{Rb}$ do que o branco, enquanto que para $\mathrm{Zn}, \mathrm{Ba}$ e $\mathrm{Fe}_{2} \mathrm{O}_{3}$ não é possível estabelecer qualquer correlação. Teores relativamente elevados de $\mathrm{Pb}$ e $\mathrm{Rb}$, respectivamente iguais a 406 e $948 \mathrm{ppm}$, foram obtidos por análise de ICP e INAA na amazonita do pegmatito (amostra AM, Tabela 2).

GEOQUímICA DO GRANITO BORRACHUDOS Análises químicas de amostras selecionadas do granito Borrachudos foram obtidas por XFR na Universidade de Bonn, Alemanha, e por XFR, INAA e ICP no Activation Laboratories, Canadá. Na Tabela 2 constam os resultados das análises de amostras representativas da zona de transição do granito Borrachudos normal (isto é, portador de feldspatos brancos) até o granito verde (amazonitizado) próximo ao contato com o pegmatito, além de uma amostra de amazonita do pegmatito.

Em termos de ambiente tectônico, os dados químicos indicam que o granito Borrachudos é do tipo anorogênico. Na Figura 3 as rochas analisadas caem no campo dos granitos intra-placa de Pearce et al. (1984) e Pearce (1996). Esse resultado está de acordo com os de outros autores, que interpretam a gênese do granito Borrachudos como relacionada à abertura do rift Espinhaço (Chemale Jr. 1987, Dossin et al. 1993, Fernandes et al. 1995b, Dussin et al. 1997).

A Tabela 2 mostra que não há diferenças composicionais significativas entre as variedades do granito branco e verde em termos dos óxidos maiores, exceto pela amostra GR-4, que é enriquecida em biotita e álcali-feldspato e empobrecida em quartzo, correspondendo, portanto, a um quartzo sienito verde. O granito é rico em sílica, com teores entre 70 e $75 \%$ em peso de $\mathrm{SiO}_{2}$, Os álcalis somam entre 8 e $9 \%$ em peso, sendo que $\mathrm{K}_{2} \mathrm{O}>\mathrm{Na}_{2} \mathrm{O}$. O teor de $\mathrm{MgO}$ é muito baixo $(\leq 0,05 \%$ em peso).

Verifica-se que os padrões de distribuição dos Elementos Terras Raras (ETR) de cinco amostras de granito (Fig. 4), normalizados aos valores de condrito C de Sun \& McDonough (1989), não apresentam variações que possam ser correlacionadas à cor. Assim, os diagramas para as amostras com feldspatos brancos (amostras GR-3, GR-1 e GR- 
Tabela 2 - Análises químicas do granito Borrachudos (em ordem crescente de intensidade da cor verde da esquerda para a direita) e de monocristal de amazonita do pegmatito (óxidos em \% peso, elementos-traço em pmm).

\begin{tabular}{|c|c|c|c|c|c|c|c|c|c|}
\hline & \multicolumn{4}{|c|}{ Granito branco } & \multicolumn{2}{|c|}{$\begin{array}{c}\text { Granito esverdeado } \\
\text { claro }\end{array}$} & \multicolumn{2}{|c|}{ Granito verde } & \multirow{2}{*}{$\begin{array}{c}\text { Amazonita do } \\
\text { pegmatito } \\
\text { AM }\end{array}$} \\
\hline & AV 001 & GR-1 & GR-2 & GR-3 & AV 002 & GR-4* & GR-5 & AV 003 & \\
\hline $\mathrm{SiO}_{2}$ & 74,31 & 74,64 & 75,02 & 70,09 & 73,60 & 61,78 & 75,78 & 74,94 & 65,08 \\
\hline $\mathrm{TiO}_{2}$ & 0,25 & 0,17 & 0,23 & 0,36 & 0,17 & 0,27 & 0,22 & 0,21 & $<0,01$ \\
\hline $\mathrm{Al}_{2} \mathrm{O}_{3}$ & 12,08 & 12,74 & 11,57 & 12,79 & 12,42 & 18,24 & 12,7 & 12,44 & 18,39 \\
\hline $\mathrm{Fe}_{2} \mathrm{O}_{3} * *$ & 2,96 & 2,08 & 2,79 & 4,43 & 2,95 & 4,39 & 2,24 & 2,71 & 0,06 \\
\hline $\mathrm{MnO}$ & 0,08 & 0,02 & 0,04 & 0,06 & 0,14 & 0,03 & 0,04 & 0,08 & $<0,01$ \\
\hline $\mathrm{MgO}$ & 0,01 & 0,02 & 0,03 & 0,05 & 0,01 & 0,03 & 0,02 & 0,02 & 0,02 \\
\hline $\mathrm{CaO}$ & 0,88 & 0,62 & 0,69 & 1,28 & 0,66 & 0,96 & 0,48 & 0,75 & 0,02 \\
\hline $\mathrm{Na}_{2} \mathrm{O}$ & 3,39 & 4,17 & 3,26 & 3,54 & 3,41 & 5,09 & 4,05 & 3,62 & 1,96 \\
\hline $\mathrm{K}_{2} \mathrm{O}$ & 4,92 & 4,56 & 4,71 & 5,20 & 5,57 & 7,06 & 4,78 & 5,12 & 13,17 \\
\hline $\mathrm{P}_{2} \mathrm{O}_{5}$ & 0,02 & 0,02 & 0,03 & 0,05 & 0,02 & 0,01 & 0,02 & 0,02 & 0,01 \\
\hline $\mathrm{PF}$ & 0,16 & 0,47 & 0,59 & 0,76 & nd & 0,83 & 0,26 & nd & 0,16 \\
\hline Total & 99,06 & 99,52 & 98,96 & 98,60 & 98,95 & 98,69 & 100,58 & 99,91 & 98,89 \\
\hline $\mathbf{B a}$ & 553 & 294 & 408 & 575 & 357 & 405 & 373 & 401 & 731 \\
\hline $\mathrm{Sr}$ & 34 & 20 & 23 & 28 & 18 & 14 & 23 & 25 & 27 \\
\hline Y & 112 & 164 & 124 & 184 & 124 & 217 & 91 & 120 & 3 \\
\hline $\mathrm{Zr}$ & 617 & 502 & 513 & 929 & 423 & 369 & 300 & 500 & 12 \\
\hline $\mathrm{Nb}$ & 45 & nd & nd & nd & 70 & nd & nd & 57 & nd \\
\hline $\mathrm{Be}$ & nd & 4 & 4 & 8 & nd & 9 & 75 & nd & 13 \\
\hline $\mathrm{Cs}$ & nd & 4,5 & 3,5 & 29,5 & nd & 300 & 160 & nd & 47,3 \\
\hline $\mathrm{Hf}$ & nd & 14,6 & 15,6 & 19,8 & nd & 16 & 12,2 & nd & $<0,5$ \\
\hline $\mathrm{Rb}$ & 190 & 259 & 207 & 238 & 642 & 888 & 685 & 891 & 949 \\
\hline $\mathrm{Sb}$ & nd & 0,4 & $<0,2$ & 0,7 & nd & 4 & 2 & nd & 0,6 \\
\hline $\mathrm{Sc}$ & 2 & 0,4 & 1 & 2 & 3 & 2,1 & 1,3 & 2 & $<0,1$ \\
\hline $\mathrm{Ta}$ & nd & 4 & 3 & 4 & nd & 57 & 15 & nd & $<1,0$ \\
\hline Th & 25 & 28,7 & $3 \mathrm{I}, 1$ & 35,6 & 54 & 53,8 & 28,6 & 74 & $<0,5$ \\
\hline $\mathrm{U}$ & 4 & 11,3 & 3,3 & 5,2 & 12 & 22,9 & 4,8 & 8 & $<0,5$ \\
\hline $\mathrm{La}$ & 122 & 90,2 & 129 & 164 & 108 & 234 & 96,5 & 154 & 0,6 \\
\hline $\mathrm{Ce}$ & 241 & 199 & 265 & 295 & 175 & 351 & 183 & 290 & $<3,0$ \\
\hline $\mathrm{Pr}$ & 23 & nd & nd & nd & 20 & nd & nd & 28 & nd \\
\hline $\mathrm{Nd}$ & 96 & 75 & 101 & 127 & 70 & 118 & 78 & 108 & $<5$ \\
\hline $\mathrm{Sm}$ & 12 & 18,7 & 22,3 & 29 & 10 & 27,6 & 17,2 & 16 & 0,1 \\
\hline $\mathrm{Eu}$ & nd & 1,0 & 1,1 & 1,3 & nd & 1,2 & 0,9 & nd & $<0,1$ \\
\hline $\mathrm{Tb}$ & nd & 3,4 & 3,2 & 4,5 & nd & 4,9 & 2,4 & nd & $<0,5$ \\
\hline $\mathrm{Yb}$ & nd & 12,7 & 10,6 & 15,3 & nd & 25,2 & 9,8 & nd & 0,3 \\
\hline $\mathrm{Lu}$ & nd & 1,8 & 1,51 & 2,35 & nd & 4,12 & 1,42 & nd & 0,05 \\
\hline $\mathrm{Pb}$ & 37 & 47 & 41 & 46 & 138 & 168 & 302 & 236 & 406 \\
\hline $\mathrm{Zn}$ & 112 & 105 & 113 & 164 & 142 & 330 & 113 & 168 & 2 \\
\hline $\mathrm{Ni}$ & 9 & nd & nd & nd & 14 & nd & nd & 10 & nd \\
\hline $\mathrm{Ga}$ & 26 & nd & nd & nd & 31 & nd & nd & 33 & nd \\
\hline As & 12 & nd & nd & nd & 18 & nd & nd & 21 & nd \\
\hline Mo & 8 & nd & nd & nd & 14 & nd & nd & 2 & nd \\
\hline
\end{tabular}

*Quartzo sienito; **Fe $\mathrm{Fe}_{2}=$ todo o Fe calculado como $\mathrm{Fe}^{3+} ; \mathrm{PF}=$ perda ao fogo; $\mathrm{Cr}, \mathrm{Co}, \mathrm{V}, \mathrm{Au}, \mathrm{Ag}, \mathrm{As}, \mathrm{Br}, \mathrm{Ir}, \mathrm{Mo}, \mathrm{Se}, \mathrm{W}$,

$\mathrm{Bi}, \mathrm{Cd}, \mathrm{Cu}=$ abaixo do limite de detecção; $\mathrm{nd}=$ não determinado

2) ficam posicionados entre os dos granitos verdes (amostras GR-4 e GR-5). Todas amostras apresentam uma forte anomalia negativa de Eu e um enriquecimento dos ETR leves em relação aos pesados. Este mesmo tipo de padrão foi encontrado por Chemale Jr. (1987) e Machado (1998) no granito Borrachudos de outras localidades. Sabe-se que, em magmas félsicos, anomalias de Eu bivalente são controladas pelos feldspatos, pois $\mathrm{Eu}^{2+}$ é compatível nestes minerais, sendo incorporado preferencialmente em plagioclásio como substituição do $\mathrm{Ca}^{2+}$ (Rollinson 1993). Embora o Eu também possa ocorrer no estado trivalente, a presença de magnetita nas rochas estudadas indica um magma mais reduzido, favorecendo, portanto, o $\mathrm{Eu}^{2+} \mathrm{em}$ detrimento ao $\mathrm{Eu}^{3+}$. A anomalia negativa de $\mathrm{Eu}$ no granito Borrachudos pode refletir um processo de gênese do magma por fusão parcial crustal, em que o $\mathrm{Eu}^{2+}$ teria ficado retido no plagioclásio cálcico restítico da fonte de magma. Quanto à observada tendência a um fracionamento dos ETR leves em relação aos pesados (Fig. 4), este pode ser causado pela permanência de piroxênios no restito da fusão parcial, pois os coeficientes de partição de piroxênios (e, em menor escala, também de hornblenda) em relação a fusões graníticas aumentam do La até o Lu (Rollinson 1993).

Os outros elementos-traço (Tabela 2) não mostram, na sua maioria,

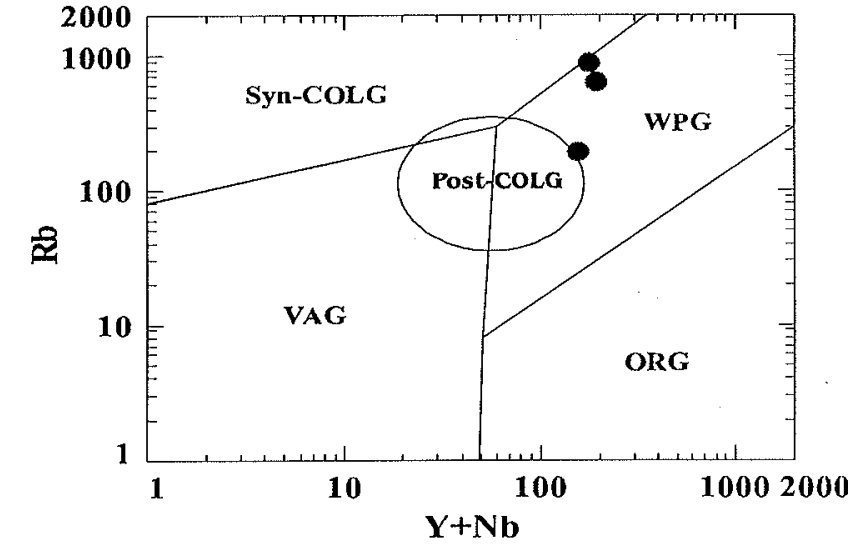

Figura 3 - Diagrana Rb vs. $Y+N b(p p m)$ de Pearce et al. (1984) e Pearce (1996) para o granito Borrachudos. Syn-COLG = granitos sin-colisionais; $W P G=$ granitos intra-placa; $V A G=$ granitos de arco vulcânico; $O R G=$ granitos das dorsais oceânicas; Post-COLG = granitos pós-colisionais. 


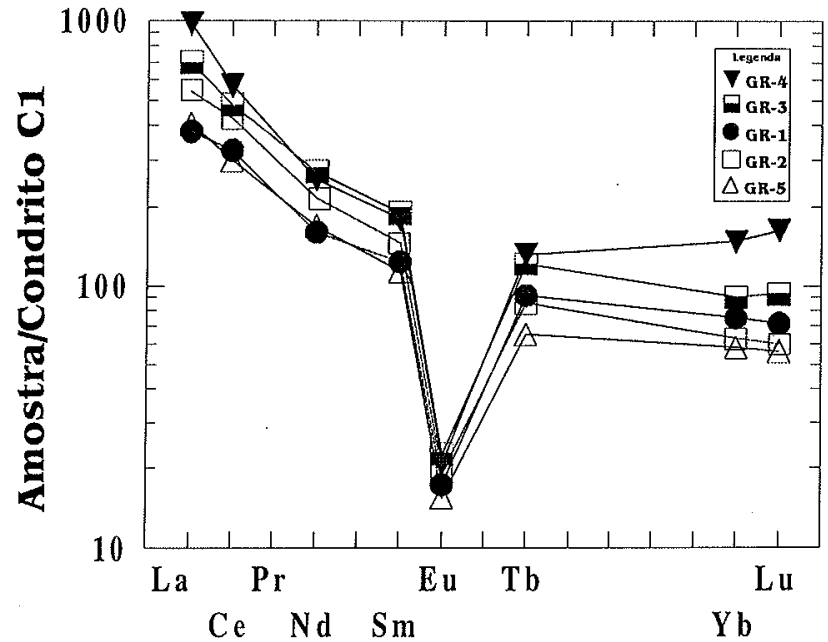

Figura 4 - Diagrama de Elementos Terras Raras do granito Borrachudos normalizado ao condrito $C_{1}$ de Sun \& McDonough (1989).

variações que possam ser relacionadas à cor. As exceções são o $\mathrm{Pb}$ e 0 $\mathrm{Rb}$, que crescem do granito normal até o granito mais verde. Verificase que os teores de $\mathrm{Pb}(\sim 40 \mathrm{ppm})$ e $\mathrm{Rb}(\sim 200 \mathrm{ppm})$ do granito normal são similares aos obtidos em amostras de outras localidades, para as quais Machado (1998, p.208) obteve valores médios de $\mathrm{Pb}=44 \mathrm{ppm}$ e $\mathrm{Rb}=179 \mathrm{ppm}$, enquanto Chemale Jr. (1987) obteve $\mathrm{Pb}=34$ e $\mathrm{Rb}=285 \mathrm{ppm}$.

DISCUSSÃO A ausência de evidências de processos de fusão parcial no granito, somada ao fato de os pegmatitos serem claramente discordantes e intrusivos (isto é, não poderiam ser pegmatitos anatécticos gerados in situ) indicam que a amazonitização deu-se no estado sólido, isto é, trata-se de um processo metassomático s.s. No intuito de determinar a causa da cor verde no granito fez-se uma análise comparativa da composição química do granito normal (com feldspato branco) e do granito verde.

Dos elementos maiores só será considerado o $\mathrm{Fe}$, o único mencionado na literatura como possível cromóforo (Petrov et al. 1993), pois a Tabela 2 mostra não haver correlação alguma entre as variações dos teores dos outros elementos e a variação da cor nos granitos. Verificase que para o $\mathrm{Fe}_{2} \mathrm{O}_{3}$, cujo teor na amazonita é insignificante $(0,06$ $\%$ em peso), não há correlação positiva com a cor verde do granito. Portanto, o Fe pode ser descartado como elemento causador da cor.

Os elementos-traço $\mathrm{Ba}, \mathrm{Rb}$ e $\mathrm{Pb}$ são mencionados na literatura (Petrov et al. 1993) como possíveis cromóforos para a amazonita. Os seus teores na amazonita do pegmatito (Tabela 2) são relativamente elevados: $\mathrm{Ba}=731 \mathrm{ppm}, \mathrm{Rb}=949 \mathrm{ppm}$ e $\mathrm{Pb}=406 \mathrm{ppm}$.

Quando se analisa o bário nas amostras de granito da Tabela 2, verifica-se que não há correlação positiva entre o seu teor e a cor verde. Além disso, o Ba determinado em amostras do Borrachudos de outros locais (858ppm: Machado 1998, p.208) é mais elevado do que em todas as amostras do presente trabalho. Portanto, estes dados indicam que o Ba não pode ser o possível elemento cromóforo.

No caso do Rb, nota-se apreciável aumento do teor médio no granito normal (224 ppm) ao verde ( $777 \mathrm{ppm}$ ) (Tabela 2 e Fig. 5). A variação chega a 4,7 vezes. O último valor é sensivelmente mais alto do que os valores encontrados para o Borrachudos de outras localidades (44ppm: Machado 1998; 34ppm: Chemale Jr. 1987). Portanto, este elemento não pode ser descartado como causador da cor verde.

As variações de $\mathrm{Pb}$ são ainda mais significativas. $\mathrm{O}$ teor de $\mathrm{Pb}$ na amazonita é de $406 \mathrm{ppm}$ e há nítida correlação positiva entre a sua concentração e a cor verde no granito $(37 \mathrm{ppm}$ no granito normal até $302 \mathrm{ppm}$ no verde, refletindo um aumento de 8,3 vezes). Este trend (Fig. 5) suporta a idéia de que o $\mathrm{Pb}$ pode ser o elemento causador da transformação dos feldspatos potássicos brancos em feldspatos verdes,

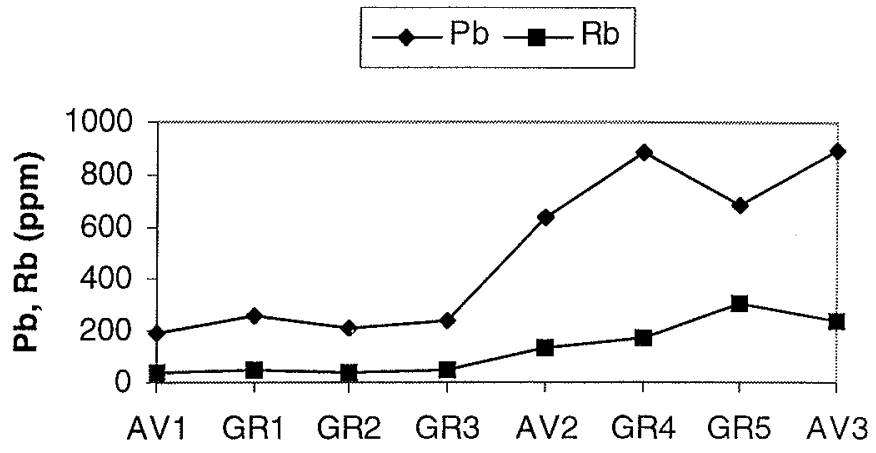

Figura 5 - Variação de $P b$ e Rb relacionada à intensidade da cor verde no granito Borrachudos: $A V_{1}, G R_{1}, G R_{2}$ e $G R_{3}$ : granito com feldspato branco; $A v_{2}$ e $G R_{4}:$ granito com feldspato verde claro; $G R_{5}$ e $A V_{3}$ : granito com feldspato verde intenso

isto é, o responsável pela amazonitização, o que está de acordo com os dados atuais da literatura (vide introdução). No entanto, o teor de $\mathrm{Pb}$ $(\sim 400 \mathrm{ppm})$ determinado na amazonita é sensivelmente menor do que se tem registrado na literatura, em que teores superiores a $1 \%$ em peso são comuns (Stevenson \& Martin 1986, Yates \& Howd 1988). Portanto, parece que mesmo quantidades pequenas de $\mathrm{Pb}$ podem causar $\mathrm{co}$ res verdes de grande intensidade em feldspatos potássicos.

CONCLUSÕES Várias hipóteses podem ser consideradas na amazonitização do granito estudado. Uma é a de que a cor verde no granito seria causada pela introdução de $\mathrm{Rb}$, que também mostra um aumento regular do granito branco ao verde (Fig. 5). Rb é mencionado na literatura como possível cromóforo na amazonita, porém Foord \& Martin (1979), que também notaram uma correlação entre o teor de $\mathrm{Rb}$ (e Cs) e a intensidade do verde, descartaram a possibilidade deste elemento ser causador da cor, porque o seu teor alcança um máximo antes do $\mathrm{Pb}$ no cristal em crescimento.

Outra hipótese é que a cor seria resultante do par $\mathrm{H}_{2} \mathrm{O}-\mathrm{Pb}$ (Hofmeister \& Rossman 1985). Neste caso há duas possibilidades. A primeira é de que o $\mathrm{Pb}$ já existia no feldspato e ter-se-ia tornado o elemento causador da cor só depois da introdução de água oriunda dos pegmatitos na estrutura do feldspato potássico. Esta possibilidade não encontra suporte no fato de os granitos com feldspato branco possuírem teores muito baixos de chumbo quando comparados com os verdes (Tabela 2, Fig. 5). A segunda possibilidade é de que a amazonitização ter-se-ia dado pela introdução tanto de $\mathrm{Pb}$ quanto de $\mathrm{H}_{2} \mathrm{O}$, em consequiência da intrusão dos pegmatitos. Levando em conta que a quantidade de $\mathrm{Pb}$ nas amostras analisadas pode ser diretamente correlacionada à intensidade da cor verde, conclui-se que esta alternativa é a mais viável, corroborando a afirmação de Hofmeister \& Rossman (1983) de que $\mathrm{Pb}$ é o único elemento que se correlaciona à cor. Segundo Doe \& Hart (1963) a adição de $\mathrm{Pb}$ em feldspatos potássicos pode ocorrer facilmente em temperaturas subsolidus sem recristalização, isto é, durante alteração do feldspato por fluidos mineralizantes ou durante metamorfismo, principalmente de contato. Doe \& Hart (1963) mostram que durante o metamorfismo de contato o halo de adição de $\mathrm{Pb}$ nos feldspatos estende-se a uma distância equivalente a aproximadamente um décimo do diâmetro da intrusão. Mais comumente, zonas de reação metassomática não excedem $10 \mathrm{~cm}$ de largura, mesmo no contato com corpos pegmatíticos de grande porte (Franz 1996). No caso estudado, verifica-se que, apesar da estrutura maciça do granito, o processo de amazonitização foi bastante eficaz, pois gerou auréolas cuja largura alcançou, localmente, um valor equivalente à largura do próprio pegmatito ( 1 a 2 metros).

Agradecimentos À FAPEMIG pelos recursos financeiros (Processo CEX-405/97). A F.A. Oliveira pela participação em trabalhos de campo preliminares, C.Dürkop (Universidade de Bonn, Alemanha) por parte das análises químicas de rocha. As análises de microssonda eletrônica foram realizadas no Instituto de Geociências da UnB. 


\section{Referências}

Bilal E., Marciano V.R.P.R.O., Fuzikawa K., Correia Neves J.M., Giret, A. 1995. Datação de monazitas do Distrito Pegmatítico de Santa Maria de Itabira, MG. In: SBG/ Núcleo MG, Simp. Geol. Minas Gerais, 8, Diamantina, Boletim, 13:46-47

Breithaupt A. 1847. Vollstïndiges Buch der Mineralogie. Band III, spezieller Teil, Dresden, Annoldi, 492p.

Cech F., Misar Z., Povondra P. 1971. A green lead-containing orthoclase. Tschermaks mineral. petrog. Mitt., 15:213-231

Chemale Jr. F. 1987 . Gênese das rochas graníticas do tipo Borrachudo. In: SBGq, Congr Bras. Geoq., 1, Porto Alegre, Anais, 1:.171-186

Doe B.R \& Hart S.R. 1963. The effect of contact metamorphism on lead in potassium feldspar near the Eldora stock, Colorado. J.Gerphys. Res., 68:3521-3530

Door J.V.N. \& Barbosa A.L.M. 1963. Geology and ore deposits of the ltabira Disctrict, Minas Gerais, Brazil. Washington, U.S. Geol. Survey Prof Paper, 341-C, $118 \mathrm{p}$.

Dossin I.A., Dossin T.M., Charvet J., Cocherie A., Rossi P. 1993. Single-zircon dating by step-wise $\mathrm{Pb}$-evaporation of Middle Proterozoic magmatism in the Espinhaço Range, southeastern São Francisco Craton (Minas Gerais, Brazil). In: SBG. Simpósio do Cráton do Sāo Francisco, 2, Salvador; Anais, 39-42

Dussin T.M. Dussin I.A., Noce C.M., Rossi P., Charvet J. 1997. Tectonic setting and origin of the Mesoproterozoic Borrachudos granites (MG, Brazil). In: SBG, SouthAmerican Symp. on Isotope Geology, Campos do Jordão, Extended Abstracts, 104 106

Fernandes M.L.S., Bilal E., Correia Neves J.M., Giret A., Costa K.V. 1995a. Estimativa da condicōes de cristalização do granito Borrachudos na região de Dores de Guanhães, condiçoes de cristalizaçấo do granito Borrachudos na regiá de Domantina, Boletim. 13:64-66

Fernandes MLS Marciano VR PR O. Oliveira R C. Correia Neves J.M. Diláscio M.V. 1995b. Granitos Borrachudos: um exemplo de granitogênese anorogênica na porção central do Estado de Minas Gerais. Geonomos, 2:23-29

Foord E.E. \& Martin, R.F. 1979. Amazonite from the Pikes Peak batholith. Mineral. Record 10:373-384

Franz G. 1996. Metasomatism at a granitic pegmatie-dunite contact in Galicia: the Franqueira occurrence of chrysoberyl (alexandrite), emerald, and phenakite: discussion. The Canad. Mineral., 34:1329-1331

Goldschmidt J.R. \& Laves F. 1954. The microcline-sanidine stability relations. Geochim. Cosmochim. Acta, 5:1.19

Hofmeister A M \& Rossman G.R. 1983. Color in feldspars. In: P.H. Ribbe (ed.) Feldspor Mineralogy, vol. 2:271-280, 2. ed., Washington, Rew. Mineral, Mineral. Soc. Am..
Hofmeister A.M. \& Rossman G.R. 1985. A spectroscopic study of irradiation coloring of amazonite: structurally hydrous, Pb-bearing feldspar. Amer: Mineral., 70:794-804 Machado G.A.A. 1998. Jazidas de Esmeralda de Capoeirana e Belmont-MG: Geologia Petrogênese e Metalogênese. Inst. de Geociências, Universidade de São Paulo, São Paulo, Tese de doutoramento, $294 \mathrm{p}$.

Marciano V.R.P.R.O., Javier Rios F., Achtschin A.B., Correia Neves J.M., Svisero D.P. 1994. Berilos de pegmatitos de Santa Maria de Itabira, Minas Gerais - Brasil. Geonomos, 2:41-50

Pearce J. 1996. Sources and settings of granitic rocks, Episodes, 19:120-125

Pearce J.A Harris N.B.W. Tindle A.G. 1984. Trace element discrimination diagrams for the tectonic interpretation of granitic rocks. J. Petrol., 25:956-983

Pedrosa-Soares A.C Dardene M A Hasui Y Castro F.D.C. Carvalho M.V.A. 1994 Mapa Geológico do Estado de Minas Gerais. Escala 1:1.000.000. Belo Horizonte Mapa Geologico do Estado de Minas Gerais. Escala

Petrov I. Mineeva R.M., Bershov L.V., Agel A. 1993. EPR of [Pb-Pb]3+ mixed valence pairs in amazonite-type microcline. Amer: Mineral., 78:500-510

Rollinson H. 1993. Using Geochemical Data: Evaluation, Presentation, Interpretation. New York, Longman Scientific \& Technical, 352p.

Schorscher H.D. 1975 . Entwicklung des polymetamorphen prakambrischen Raumes Itabira, Minas Gerais, Brasilien. Ruprecht-Karl-Universität, Heidelberg, Alemanha, Tese de doutoramento, 302p.

Stevenson R.K. \& Martin R.F. 1986. Implications of the presence of amazonite in the Broken Hill and Geco metamorphosed sulfide deposits. Can. Min., 24:729-745

Sun S. -s. McDonough W. F. 1989. Chemical and isotopic systematics of oceanic basalts: implications for mantle composition and processes. In A.D. Saunders \& M.J. Norry (eds.) Magmatism in the Ocean Basins, London, Geological Society Special Publication, 42:313-345

Yates M. A \& Howd F.H. 1988. Contact metamorphism of the Blue Hill $\mathrm{Zn}-\mathrm{Pb}$-Cu deposit Hancock Co Maine and the developmente of perthite amazonite. In: Geol. Soc. Am., Annual Meeting, 23, Portland.. Abstractss, 20(1):80

Manuscrito A-1122

Recebido em 23 de setembro de 1999

Revisão dos autores em 15 de abril de 2000 Revisão aceita em 25 de setembio de 2000 